\title{
Visualizing the Mass and Width Spectrum of Unstable Particles
}

\author{
N.L. Harshman \\ Formerly: Department of Physics and Astronomy \\ Rice University \\ Houston, TX \\ Currently: Department of Computer Science, Audio Technology and Physics \\ American University \\ Washington, DC
}

August 22, 2018

\begin{abstract}
Several graphical representations of the mass and width spectrum of unstable subatomic particles are presented. Such plots are useful tools for introducing students to the particle zoo and provide students an alternate way to organize conceptually what can seem like an overwhelming amount of data. In particular, such graphs highlight phenomenological features of unstable particles characteristic of different energy and time scales.
\end{abstract}

\section{Introduction}

When confronting the vast array of subatomic particles detected and created in particle physics experiments, new students (and old researchers) may become overwhelmed with the 
volume of descriptive information. The purpose of this note is to present a visual tool to supplement other schemes used to organize the diversity of unstable particles. The idea is simple: what does a plot of width versus mass of subatomic particles look like and what information does it convey about the kinematics and dynamics of these particles? I hope that instructors of particle physics may find it useful when introducing new visitors to the particle zoo and that practitioners may find an alternate perspective stimulating.

The most useful feature of these graphs is not that they summarize information about the spectrum or structure of unstable particles, but that they highlight the distinctions between the phenomenological signatures of unstable particles at different energy and time scales. These graphs provide a natural way of grouping together particles, not by their physical content, but by how they are produced, how they decay, and how their characteristic parameters are measured. This alternate taxonomy distinguishes three classes of unstable particles: decaying states, which decay via the weak interaction and whose exponential decay rate can be measured, resonance states, which decay primarily through the strong interaction and are measured as features in the cross section, and a middle class of "platypus" states, whose instability parameters require more subtle measurements to determine. Also, these graphs can be used to call attention to how the limitations and prejudices of past experiments affect what is known about the mass and width spectrum of particles.

\section{Which Unstable Particles?}

Figure 1 depicts the mass $M$ and width $\Gamma$ of 139 unstable particles, with mass plotted logarithmically on the horizontal axis and width plotted logarithmically vertically. The shape of each plotted point indicates the type of particle it is (gauge boson, e.g.) and for the hadrons the style (black, gray, or hollow) indicates some information about the quark content.

The data for the 139 unstable particles come from the 2002 edition of The Review of Particle Physics [1, and in particular the list of well-known, reasonably well-measured unstable particles from the "Summary Tables of Particle Properties" therein. Not every particle in the "Summary Table" has been included; only those particles found in the file [2] that the Particle Data Group tabulates, of the mass and width data, for use in Monte Carlo event generators and detector simulators. For unstable particles whose lifetimes $\tau$ are quoted in the Review [1] and not their widths, the width values in the Monte Carlo file are found using the Weisskopf-Wigner relation $\Gamma=\hbar / \tau$ (more will be said about this later).

The list of particles from the Monte Carlo file [2] has been modified and applied in the following way in Fig. 1 and subsequent figures:

1. The stable particles, the proton, electron, photon and neutrinos, are excluded. 
2. The nearly-stable neutron is neglected for reasons of scale.

3. The Monte Carlo file includes some particles for which only an upper bound of the width has been measured. They have been excluded. Examples include some light unflavored meson resonances like the $f_{0}(980)$, other meson resonances such as the $D_{s}^{* \pm}$ and $\chi_{b 0}(1 P)$, and a few baryon resonances like the $\Sigma_{c}(2520)^{+}$.

4. The top quark, not truly an independent particle like the others in the list, is not included.

5. The symbol plotted for a particle also represents its antiparticle, except for the neutral K-mesons. For these, the mass eigenstates $K_{S}^{0}$ and $K_{L}^{0}$ are plotted instead of the flavor eigenstates $K^{0}$ and $\bar{K}^{0}$.

6. A single symbol is plotted for all different charge-species of a baryon unless different masses for different charges have been measured. For example, each point representing a $\Delta$ baryon represents all four charge species $\{++,+, 0,-\}$ corresponding to quark contents $\{u u u, u u d, u d d, d d d\}$.

Then what unstable particles are included? The weak gauge bosons $\mathrm{W}$ and $\mathrm{Z}$ are at the high energy extreme and the muon is at the low energy extreme. The other unstable lepton, the tau, is in the middle, along with a host of hadrons made up of five out of the six quarks: up, down, strange, charm and bottom. While the gauge bosons and leptons are to our best knowledge structureless; the hadrons are composite. Subsequent references to particles refer just to this set of well-established, well-measured unstable particles, and therefore should not be taken to refer to all possible particles that have or have not been observed or theorized.

Looking at Figure 1, it may be tempting to ask if there is a functional dependence of the width on the mass. In principle the widths of unstable particles are calculable from the masses of the quarks and leptons and other Standard Model parameters, although in practice such calculations are difficult or impossible, especially for hadrons. For the purposes of this article, the width and mass are considered independent phenomenological parameters to be determined from experiment.

Figure 1 does make apparent the general trend of increasing width with increasing mass, which is explained by phase space effects. The decay rate is roughly proportional to the phase space of the decay products and the more massive the unstable particle, the more decay channels are available.

To better elucidate the properties of the scattered distribution in Figure 1, several other partitions or sections of the data are included below. Figure 2 plots the masses of the 139 unstable particles in order of increasing mass, i.e. in rank order from the least massive to 
the most massive. Figure 3 is similar, except it plots them in rank order of increasing width. Figure 4 plots the unitless ratio of width to mass in increasing order. These different graphs give further clues about the structure of the mass and width spectrum and how to identify phenomenologically-similar groups of unstable particles.

\section{Mass Spectrum}

A striking feature of Figure 1 is that while the widths run over a range of 18 orders of magnitude, the masses are constrained within three decades, with most of them between $1 \mathrm{GeV}$ and $10 \mathrm{GeV}$. This fact says more about the types of experiments that have been performed than about the "essential nature" of the mass spectrum. Far more particle searches have focussed on this energy range for a variety of historical and practical reasons.

Another perspective on the mass spectrum can be gained from Figure 2. Qualitatively, gaps in the plot indicate how quark composition affects (and effects) the mass spectrum of hadrons. For example, at the low end, twelve of the first thirteen points are mesons consisting of the three light quarks: up, down and strange. The non-meson among the thirteen is the muon, the lightest unstable particle, which historically was mistaken for a meson. The twelve mesons have masses less than the lightest (undepicted) baryons, the proton and neutron at about $940 \mathrm{MeV}$. There is a gap between the first three points, which represent the muon, $\pi^{0}$ and $\pi^{ \pm}$, and the next set, which include the lightest mesons composed of strange quarks, the $K^{ \pm}, K_{S}^{0}, K_{L}^{0}$ and $\eta$.

At the high end of the mass spectrum, except for the gauge bosons, the points are dominated by hadrons containing heavy quarks. The lightest hadron containing a charm quark, the $D^{0}$, is eighty-fifth on the list with a mass of $1865 \mathrm{MeV}$ and the lightest hadrons containing a bottom quark are the B-mesons $B^{ \pm}$and $B^{0}$ with ranks of 127 and 128 and masses of $5279 \mathrm{MeV}$. Similar to the strange quark mass threshold jump seen at the lower energies, here there are slight jumps at 117, the lightest particle with two charm quarks $\eta_{c}$, at 127 , the lightest particle with one bottom quark $B^{ \pm}$, and at 132 , the lightest particle with two bottom quarks $\Upsilon(1 S)$.

The fact that above number 109 all points represent hadrons containing charm or bottom quarks does not mean that unstable particles containing only light quarks are not found in this mass range. It only indicates that such resonances have not been the focus of experimental searches in that energy range. Also, at such high energies production of light mesons is so copious that wide, light-meson resonances get lost in the background.

Finally, the true heavy-weights, the gauge bosons, with masses near $100 \mathrm{GeV}$, stand alone at the far sides of Figure 1 and Figure 2. Their isolation is again an artifact of experimental particle physics history, not of some fundamental nature of the mass spectrum of particles. 
To make precise measurements of these particles entailed building experiments with far more energy and at a far greater cost. To better explore the physics at this scale and to search for potentially heavier particles such as the Higgs boson will require the next generation of particle accelerators.

\section{Width Spectrum}

Unlike the mass spectrum, the width spectrum (i.e., the plot of the widths of the 139 unstable particles in increasing order) depicted in Figure 3 is not as dependent on the energy scales of experiments that have been performed. Figure 3 spans many decades of the width, and even the most massive particles can have very small or very large widths. For example, the $\Lambda_{b}$ baryon, with mass and width $\left(5.62 \mathrm{GeV}, 5.36 \times 10^{-13} \mathrm{GeV}\right)$, has a mass rank of 130 out of 139 , but a width rank of 15 . It is considered unlikely that we will discover any more stable or extremely long-lived subatomic particles, so future discoveries will likely either fit into this graph or, perhaps for new ultra-massive particles like the Higgs boson, be appended to the end, beyond the gauge bosons. Therefore the shape of this graph is unlikely to change much as unstable particles are added, whereas new particle discoveries will probably smooth out the high energy, quark-mass threshold gaps in the mass spectrum Figure 2.

Looking at Figure 3, it seems natural to roughly break the graph into three parts: particles with widths $\Gamma<10^{-8} \mathrm{MeV}, 10^{-5} \mathrm{MeV}<\Gamma<10 \mathrm{MeV}$, and $\Gamma>10 \mathrm{MeV}$. This division becomes even more sensible when Figure 4, which lists the particles in order of the widthto-mass ratio $\Gamma / M$, is considered. The first 40 particles in both Figures 3 and 4 have nearly the same order; only after that is there a substantial reshuffling. In Figure 4 a heuristic division between nearly the same three classes of particles could be made at $\Gamma / M<10^{-10}$, $10^{-8}<\Gamma / M<10^{-2.5}$ and $\Gamma / M>10^{-2.5}$.

\subsection{First Class: Decaying States}

The interesting thing about these classes is that they have physical significance. In Figure 1, this first class of particles is the arc of leptons, mesons and baryons along the lower part of the graph. From the point of view of fundamental interactions, what these particles have in common is that they decay via the weak interaction, and consequently they are long-lived.

Long-lived is of course a relative term, but widths $\Gamma<10^{-8} \mathrm{MeV}$ correspond to lifetimes $\tau>10^{-14} \mathrm{~s}$. In terms of phenomenology, that means for particles in this first class the exponential decay rate can be measured directly. This measurement involves finding the distance traveled between the production location and the decay vertex. This information, combined with kinematic information of mass, momentum, energy and/or speed, can be 
used to find the time-of-flight in the rest frame of the particle. A histogram of all the time-of-flights for a certain particle type can be fit to an exponential to get the lifetime.

Long life makes this first class seem like the most "particle-like" of all the unstable particles. In many calculations they can be approximated as stable because they are stable with respect to the much shorter time scales dictated by the strong and electromagnetic interactions. Their width/mass ratios $\Gamma / M$ are so small that any mass uncertainty can be neglected in kinematic calculations.

\subsection{Third Class: Resonances}

In contrast, particles in the third class have widths $\Gamma>10 \mathrm{MeV}$, corresponding to particles with lifetimes $\tau<10^{-22}$, and to a width-to-mass ratio $\Gamma / M$ greater than a part in a thousand. Because of this mass uncertainty, they can be produced in experiments with energies substantially lower or higher than their central (quoted) mass value. This class of particles decays primarily through strong interactions and the vast lifetime difference separating them from the first class is attributable to the strength of the strong interaction and the massiveness of the weak gauge bosons.

It may seem unjustified to consider these short-lived unstable particles as the same kind of object as long-lived unstable particles, so vast are the differences in their instability parameters. The distinction between these two classes is sometimes codified in the language of particle physics: the long-lived particles are called decaying states and the short-lived are called resonances. This distinction arises because of the different ways that decaying states and resonances are observed experimentally.

Particle resonances are detected as rapid variations (usually peaks) in the cross section. As the center-of-mass energy of a collision is scanned over some range, there may appear an enhancement of the elastic cross section or the cross section into a particular set of inelastic channels. After extracting the background and accounting for uncertainties in the preparation and detection apparatuses and other effects (such as radiative corrections), the resonant cross section $\sigma_{R}$ as a function of center-of-mass energy (or center-of-mass energy squared s) can be extracted. This process can become more complicated if there are multiple resonances in the same energy region, interfering resonances, or background-resonance interference.

Neglecting these complications, the resonance cross section $\sigma_{R}$ (or resonance lineshape or linewidth) can be fit to a theoretical function and the mass and width extracted. Typically, the function used is the Breit-Wigner or Lorentzian function, which can be parameterized in terms of mass and width as

$$
\sigma(\mathrm{s}) \propto\left|\frac{1}{\mathrm{~s}-(M-i \Gamma / 2)^{2}}\right|^{2},
$$


or by several other parameterizations. This functional form for the resonance lineshape can be derived by associating the resonance to a pole in the scattering S-matrix [3, 4. Alternate functions exist; for example, in perturbative quantum field theory, the on-mass shell renormalization scheme leads to a different definition for mass and width (for a discussion of this as applied to the Z-boson, see [5]). For some resonances, such as the $\Delta$ baryons, the Review [1] cites values for mass and width corresponding to both the Breit-Wigner and perturbation theory definitions.

To measure the resonance cross section accurately and to extract a value for mass and width, whichever function and parameterization are used, requires experimental energy resolution precise enough to trace out the lineshape. Practically, this means the energy resolution should be smaller than the ratio $\Gamma / M$ for that particular resonance. As a result, the lineshapes of particles in the first class, with $\Gamma / M<10^{-10}$, cannot be measured in this way. Measurements of width and lifetime are physically very distinct processes that apply to phenomena at very different energy scales.

The width and lifetime of a particle are related theoretically by the Weisskopf-Wigner formula $\tau=\hbar / \Gamma$, which was originally proposed in the context of atomic electronic lineshapes [6]. This relation is considered so standard as to appear to be a definition or identity or direct consequence of the uncertainty principle, but to the best knowledge of this author, this relationship has never been verified experimentally in the regime of subatomic physics [7]. The Wigner-Weisskopf relation between the width and decay rate or inverse lifetime $\Gamma / \hbar$ is derived as an approximation in non-relativistic scattering in many textbooks (for example [3, 11]), and can be proven identically in the non-relativistic and relativistic case for the mathematical object called a Gamow vector [12].

\subsection{Middle Class: Troublemakers}

The middle class of particles on the chart, with widths roughly between $10^{-5} \mathrm{MeV}<\Gamma<10$ $\mathrm{MeV}$ and width-to-mass ratios $10^{-8}<\Gamma / M<10^{-2.5}$, are awkwardly placed from an experimental point of view. Their lifetimes are so short that direct measurement of exponential decay is extremely difficult or impossible; their widths are so narrow that few experiments have the energy resolution to accurately trace their lineshape. While the middle class is easily distinguishable from the decaying states in Figure 1, they blend into the bottom of the resonance states. There is no clear gap characteristic of the difference in strength between electromagnetically- and strongly-decaying states.

Physically, what do states in this middle class have in common? Some of these states have substantial branching ratios into electromagnetic decay channels like the $\pi^{0}$ and $\Sigma^{0}$ and do not decay via the strong interaction. Some, like the $D^{*}(2020)^{ \pm}$are barely above the energy threshold of for their primary decay channels and their decays are therefore 
phase-space suppressed. All the mesons in this class (except the $\left.D^{*}(2020)^{ \pm}\right)$are unflavored, i.e. composed of quarks and antiquarks of the same flavor (or superpositions of same-flavor quark/antiquark pairs) and are not energetic enough to decay into strongly-favored channels.

Neither decaying states nor resonances by the definitions described above, how are the widths of these states measured? Below, the experimental determinations of some of these particles' widths are described to give a flavor for some of the other tools at the disposal of particle physicists.

- With a lifetime of $(8.4 \pm 0.6) \times 10^{-17} \mathrm{~s}$, the exponential decay rate of the $\pi^{0}$ is very hard to measure. The $\pi^{0}$ decays almost exclusively into a pair of photons via the electromagnetic interaction. A precision experiment by Atherton, et al. [13, fit an exponential to three data points extracted from the distance between the production and the decay of the $\pi^{0}$ into two photons. The measurement agrees (within error) with another method used to measure widths and lifetimes: the Primakoff effect [14. Bombarding heavy nuclei with gamma rays, an interaction between the incoming photon and a virtual photon can produce the $\pi^{0}$; this process is called photo-production. One can measure the cross section for this process and relate it theoretically via the transition amplitude to the partial width or decay rate $\Gamma_{2 \gamma}$ for the process $\pi^{0} \rightarrow \gamma \gamma$. From this, the total width is determined by dividing the partial width $\Gamma_{2 \gamma}$ by the independently-measurable branching ratio $B_{2 \gamma}$ into the two photon decay channel, i.e., $\Gamma=\Gamma_{2 \gamma} / B_{2 \gamma}$.

- The Primakoff effect has also been used to measure the lifetime of the $\Sigma^{0}$ baryon as $(7.4 \pm 0.7) \times 10^{-20}$ s. The $\Sigma^{0}$ decays almost exclusively to $\Lambda \gamma$.

- For the $c \bar{c}$ resonances $J / \psi$ and $\psi(2 S)$ and the $b \bar{b}$ resonances $\Upsilon(1 S), \Upsilon(2 S)$ and $\Upsilon(3 S)$ the partial width $\Gamma_{e e}$ is extracted from the integrated cross section, for example, in the elastic process $e^{+} e^{-} \rightarrow J / \psi \rightarrow e^{+} e^{-}$. Then the ratio of the elastic cross section to the total cross section independently provides the branching ratio $B_{e e}$. The total width (as above) is $\Gamma=\Gamma_{e e} / B_{e e}$.

- Both the above method and the Primakoff effect have been applied to the $\eta$ meson giving consistent results and a value for the width of $\Gamma=1.18 \pm 0.11 \mathrm{keV}$.

- Finally, the $\mathrm{D}_{0}^{*}(2010)^{ \pm}$, with a width of $\Gamma=96 \pm 4 \pm 22 \mathrm{keV}$, has a mass just above the threshold for its main decay channels $D^{0} \pi^{ \pm}$and $D^{ \pm} \pi^{0}$. Its width cannot be measured directly, but can be extracted from fitting to simulations of the the energy distribution of decay products [15. 


\section{$5 \quad$ Summary and Further Considerations}

The mass-width spectrum in Figure 1 does not reveal as much information about unstable particles as Hertzsprung-Russell diagrams reveal about stellar composition and evolution [16]. These graphs do not contribute to finding a perturbative, renormalizable, elegant theory for predicting masses and widths of hadrons based on standard model parameters. Nonetheless, Figure 1 and subsequent partitions of it do show some structures that correspond to significant phenomenological features. As a result, I think these graphs provide an excellent tool (or at least a starting point) for instructing students of particle physics about a host of physical phenomena and experimental procedures. A partial list of these features or ideas would include:

- Relative strengths of the fundamental interactions.

- Connection of the transition amplitude and scattering matrix to measurable quantities.

- Experimental measurement of exponential decay.

- Resonance production and decay.

- Cross section measurements.

- Phase space dependence of width and decay rate.

Additionally, not just the viewing of these graphs, but the production of these kinds of plots would be an excellent exercise for students, undergraduate or graduate. While familiarizing themselves with the particle zoo, they could also practice using graphing and data management software. As an example of other kinds of plots possible, Fig. 5 depicts the width versus mass of just the mesons on a linear scale.

Finally, visualizing the mass and width spectrum of unstable particles with such graphs makes the task of conceptually organizing the physical data of hundreds of unstable particles a little easier. It provides an alternate way to group them into phenomenologicallymeaningful families, complimenting standard organization schemes according to flavor and/or quark content. Many physicists have some biological, taxonomical part of their brains to which I hope these graphs appeal. 


\section{References}

[1] K. Hagiwara et al., "The Review of Particle Physics," Phys. Rev. D 66, 010001 1-974 (2002).

[2] http://pdg.lbl.gov/rpp/mcdata/mass_width_02.mc

[3] For example, see M. L. Goldberger, K. M. Watson, Collision Theory (Wiley, New York, 1964), chap. 8.

[4] See also, Arno Bohm, "Quantum Mechanics: Foundations and Applications," (Springer, New York, 1994), 3rd ed., chap. 18.

[5] Arno R. Bohm and N.L. Harshman, "On the mass and width of the Z-boson and other relativistic resonances," Nucl. Phys. B 581, 91-115 (2000).

[6] V. Weisskopf and E. P. Wigner, "Berechnung der natülichen Linienbreite auf Grund der Diracsichen Licht theorie," Z. f. Physik 63, 54-73 (1930) and "Uber die natürlich Linienbreite in der Strahlung des harmonischen Oszillators," 65, 18-27 (1930).

[7] The Wigner-Weisskopf relation has been verified some time ago for some nuclear unstable states [8, 9] using the Mössbauer effect to achieve the required energy resolution and more recently for one atomic unstable state [10] using ultra-cold atoms for the same purpose.

[8] For example, see H. Frauenfelder, The Mössbauer Effect (W.A. Benjamin, New York, 1962), pp. 50-51.

[9] P.A. DeYong, P.L. Jolivette, and N. Rouze, "Experimental verification of the Heisenberg uncertainty principle - an advanced undergraduate laboratory," Am. J. Phys. 61 (6), 560-563 (1993).

[10] U. Volz, M. Majerus, H. Liebel, A. Schmitt, and H. Schmoranzer, "Precision Lifetime Measurements on NaI $3 p^{2} P_{1 / 2}$ and $3 p^{2} P 3 / 2$ by Beam-Gas-Laser Spectroscopy," Phys. Rev. Lett. 76, 2862-2865 (1996); C.W. Oates, K.R. Vogel, and J.L. Hall, "High Precision Linewidth Measurement of Laser-Cooled Atoms: Resolution of the $\mathrm{Na} 3 p^{2} P_{3 / 2}$ Lifetime Discrepancy," Phys. Rev. Lett. 76, 2866-2869 (1996).

[11] For a more elementary discussion than that in [3] based on time-dependent perturbation theory, see Eugen Merzbacher, Quantum Mechanics (Wiley, New York, 1970), 2nd ed., chap. 18, which includes the quotation "To obtain nonreversing transitions and a 
progressive depletion of the initial state it is essential that the discrete initial state be coupled to a very large number of states with similar frequencies. However, the fact remains that the exponential decay law, for which we have so much empirical support in radioactive processes, is not a rigorous consequence of quantum mechanics but the result of somewhat delicate approximations," pp. 484-485.

[12] Gamow was the first to use eigenfunctions of the Hamiltonian with complex energy for a heuristic description of unstable states in G. Gamow, "Zur Quantentheorie der Atomkernes," Z. Phys. 51, 204-218 (1928). For a modern non-relativistic treatment, see chap. 21 of 4]; for a relativistic discussion, see [5]; also, A. Bohm, N.L. Harshman and H. Walther, "Relating the Lorentzian and exponential: Fermi's approximation, the Fourier transform, and causality," Phys. Rev. A 66, 012107 1-11 (2002).

[13] H.W. Atherton et al., "Direct measurement of the lifetime of the neutral pion," Phys. Lett. 15B, 81-84 (1985).

[14] H. Primakoff, "Photo-production of neutral mesons in nuclear electric fields and the mean life of the neutral meson," Phys. Rev. 81, 899 (1951).

[15] A. Anastassov et al. (CLEO Collaboration), "First measurement of $\Gamma\left(D^{*+}\right)$ and precision measurement of $m_{D^{*+}}-m_{D^{0}}, "$ Phys. Rev. D 65, 032003 1-11 (2002).

[16] M. Zeilik, S. A. Gregory, E. v. P. Smith, Introductory Astronomy and Astrophysics, Third Edition, (Harcourt Brace Javanovich, Fort Worth, 1992), p. 249-267. 


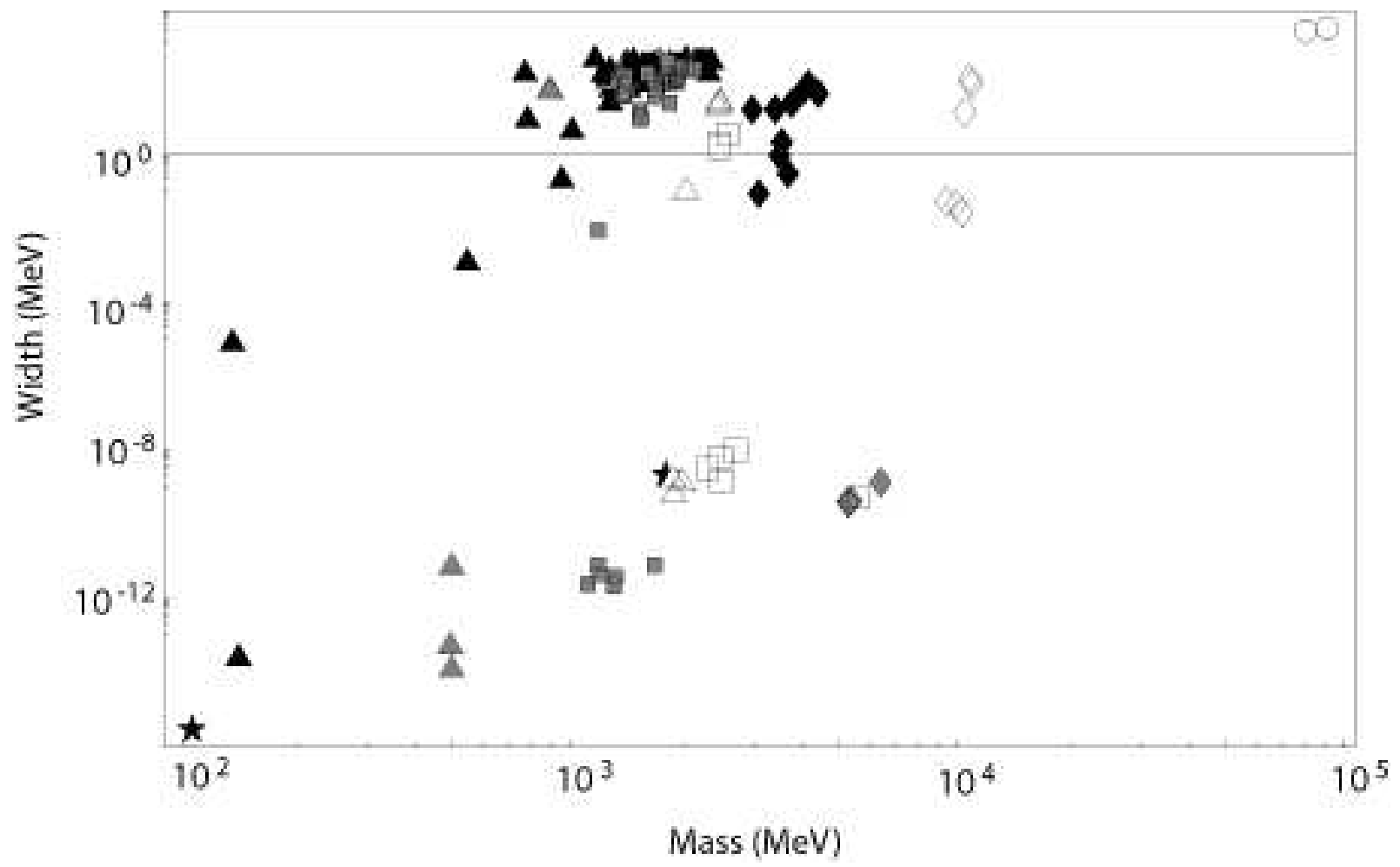

Fig. 1. Log-log plot of Mass/MeV versus Width/MeV. Choice of 139 unstable particles plotted described in text. Key: hollow circles-gauge bosons; black stars-leptons; black triangles - light unflavored mesons; gray triangles - strange mesons; hollow triangles - flavored charmed mesons (including charmed-strange mesons); black diamonds - unflavored charmed $(c \bar{c})$ mesons; gray diamonds - flavored bottom mesons (including bottom-strange and bottom-

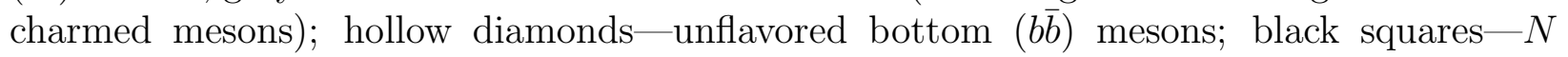
and $\Delta$ baryons; gray squares - strange baryons (including $\Lambda, \Sigma, \Xi$ and $\Omega$ baryons); hollow squares - charmed and bottom baryons. 


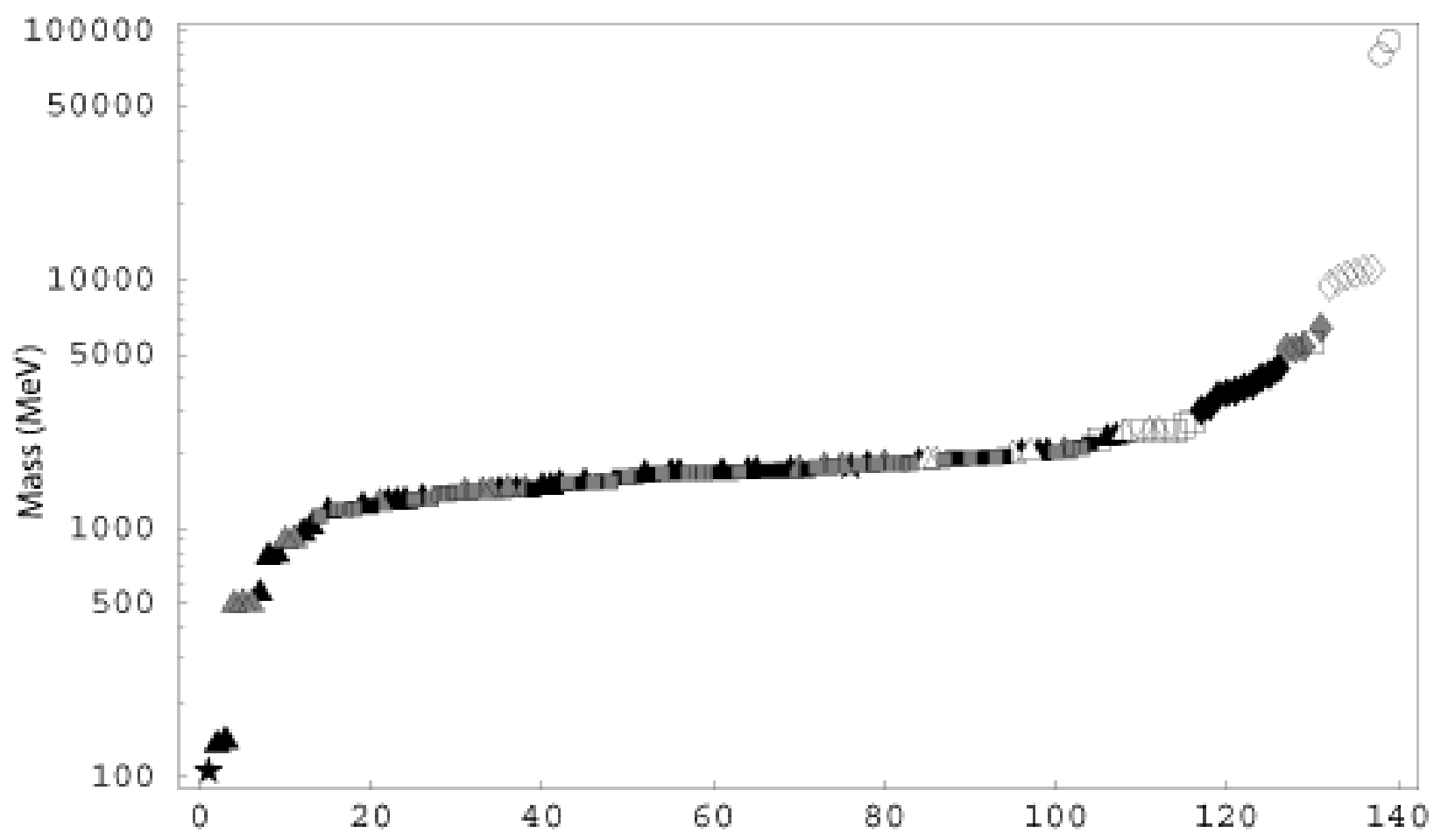

Fig. 2. Log-linear plot of Mass/MeV versus particle rank in order of increasing mass (out of 139 selected particles). Key is same as in Fig. 1. 


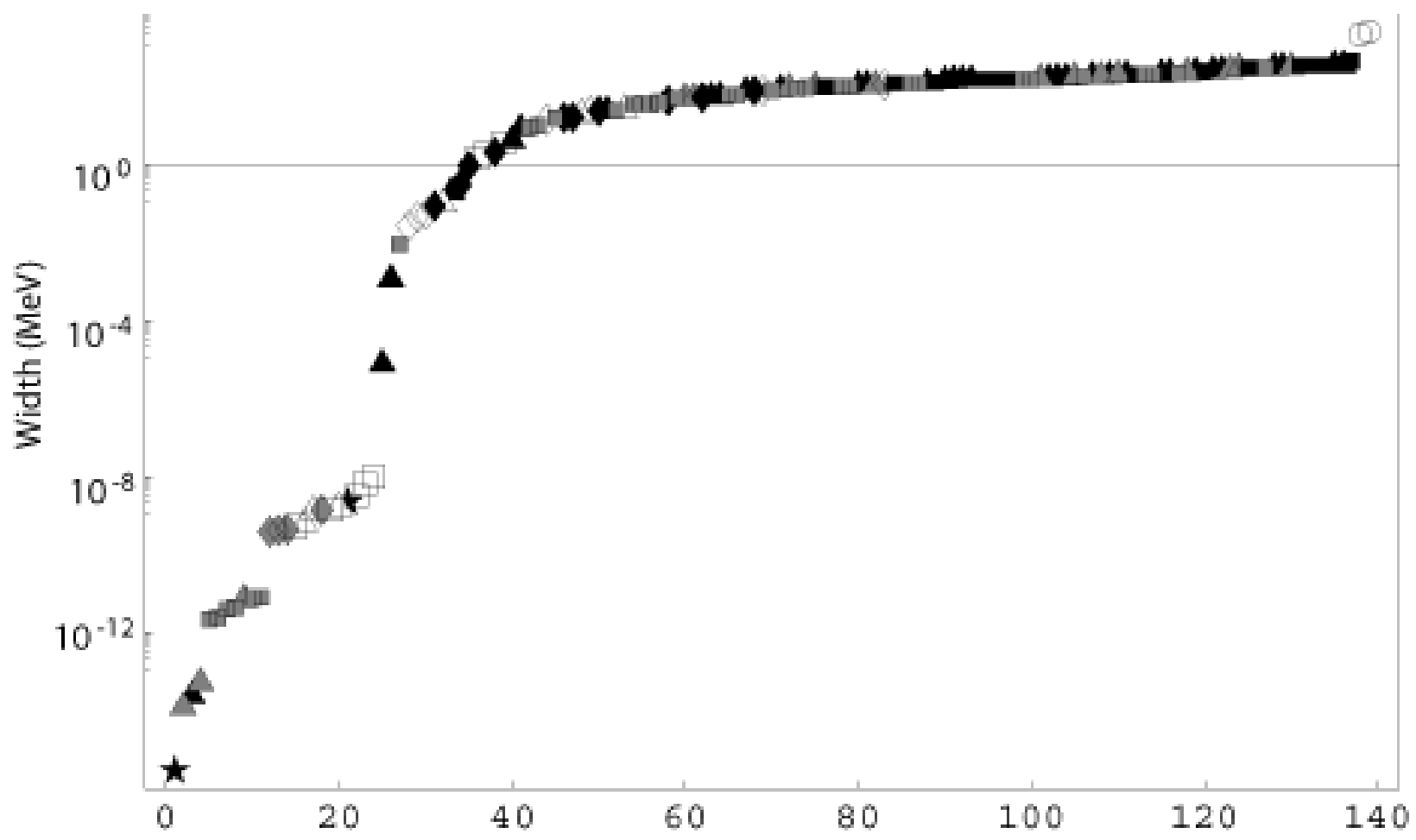

Fig. 3. Log-linear plot of Width/MeV versus particle rank in order of increasing width (out of 139 selected particles). Key is same as in Fig. 1. 


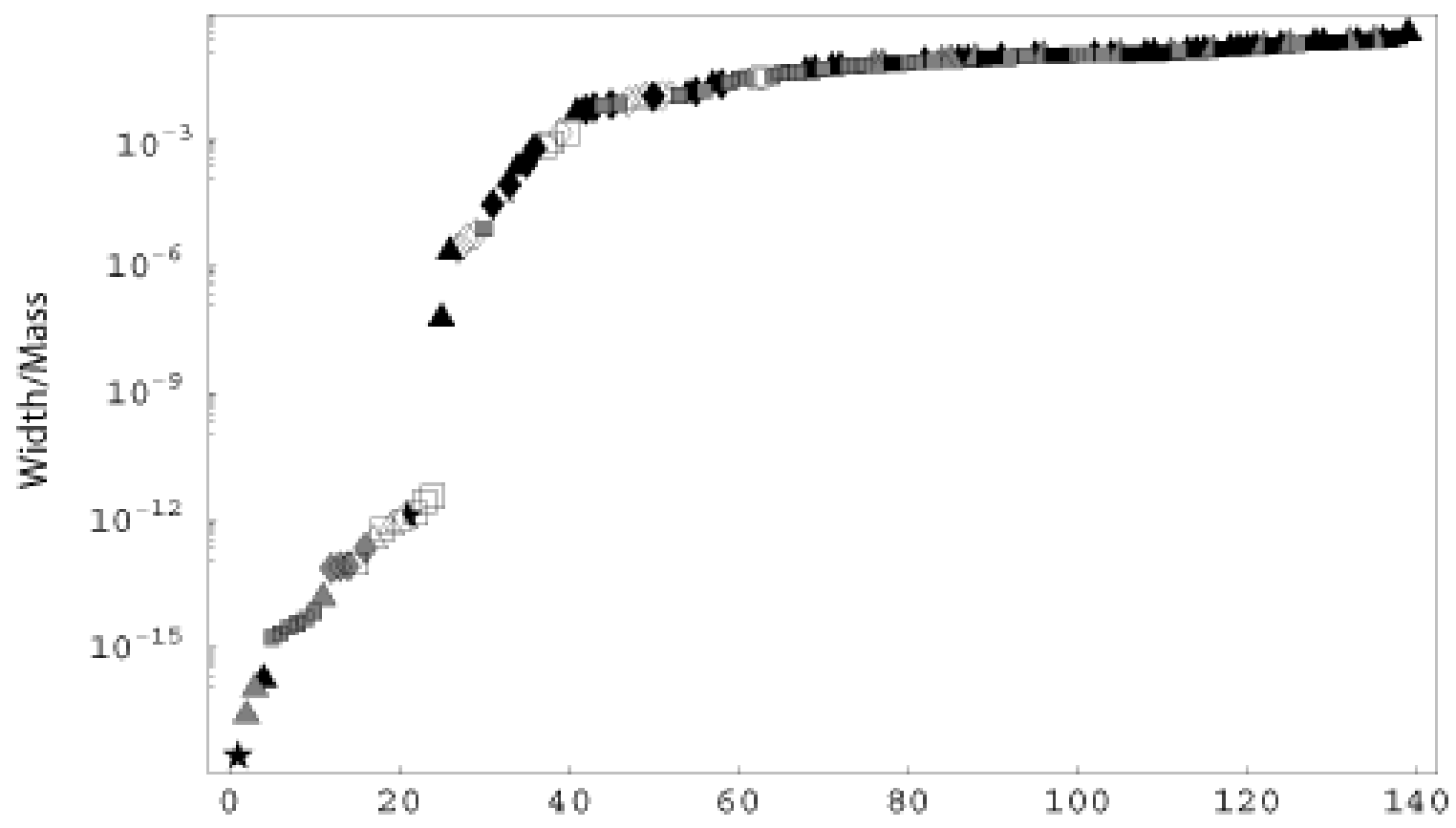

Fig. 4. Log-linear plot of width-to-mass ratio $\Gamma / M$ versus rank in order of increasing widthto-mass ratio (out of 139 selected particles). Key is same as in Fig. 1. 


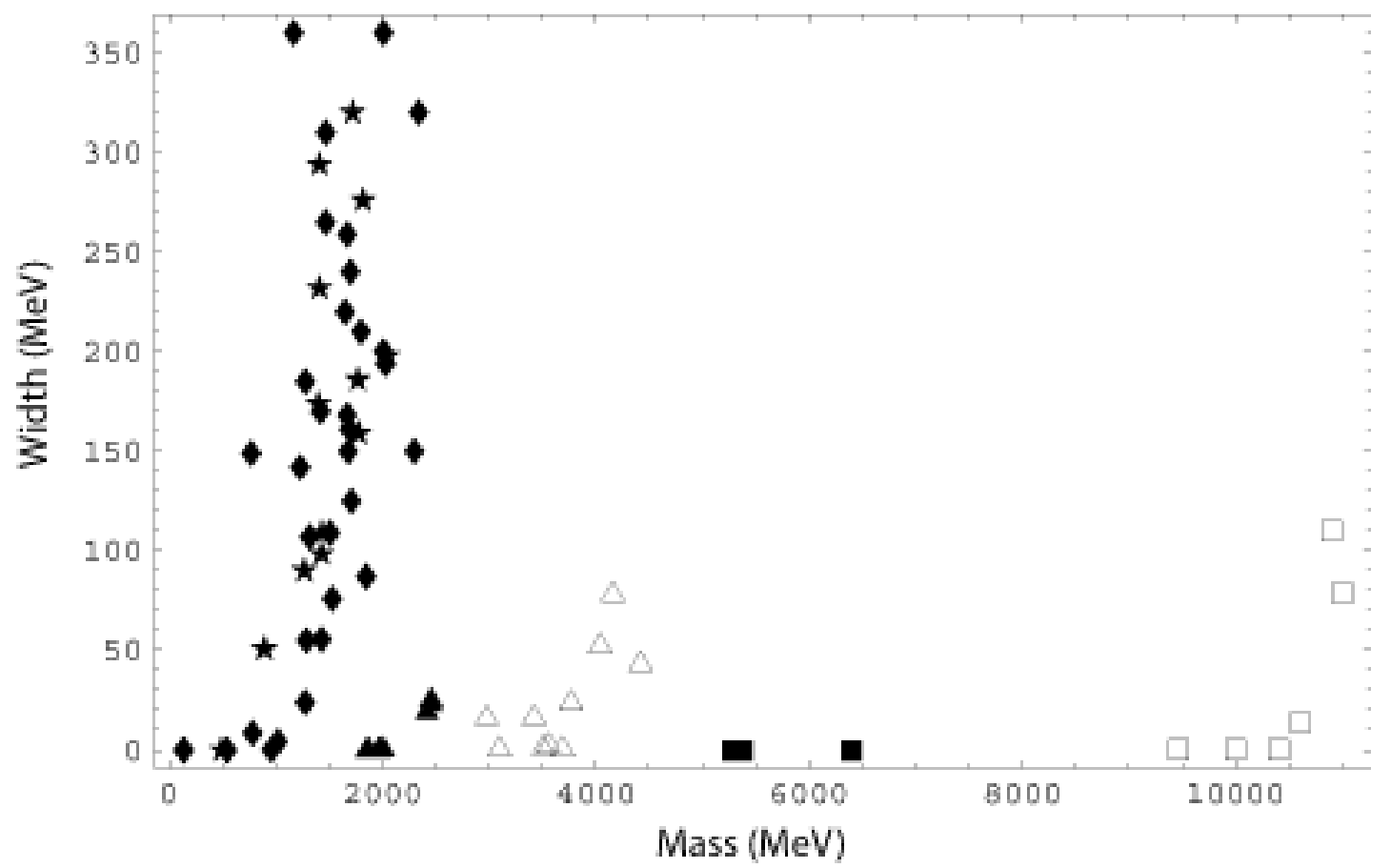

Fig. 5. Linear plot of width versus mass of unstable mesons. Key: black diamonds - light, unflavored mesons, black stars - strange mesons; black triangles - charm-flavored mesons (including charmed/strange-flavored mesons); hollow triangles - $c \bar{c}$-mesons; black squaresbottom-flavored mesons (including bottom/strange- and bottom/charmed-flavored mesons); hollow squares - $b \bar{b}$-mesons. 